\title{
Quantum Switch for Continuous Variable Teleportation
}

\author{
Junxiang Zhang, Changde Xie, Kunchi Peng \\ Institute of Opto-electronics, Shanxi University \\ The Key Laboratory of quantum optics, Ministry of Education, China \\ Shanxi, Taiyuan 030006, P.R.China
}

Tel:+86-351-7010688, Fax:+86-351-7011500

Email: kcpeng@sxu.edu.cn

\begin{abstract}
A novel quantum switch for continuous variables teleportation is proposed. Two pairs of EPR beams with identical frequency and constant phase relation are composed on two beamsplitters to produce two pairs of conditional entangled beams, two of which are sent to two sending stations(Alices) and others to two receiving stations(bobs). The EPR entanglement initionally results from two-mode quadrature squeezed state light. Converting the squeezed component of one of EPR sources between amplitude and phase, the input quantum state at a sender will be reproduced at two receivers in turn. The switching system manipulated by squeezed state light might be developed as a practical quantum switch device for the communication and teleportation of quantum information.
\end{abstract}

PACS numbers: 03.67.HK. 42.50.Dv.

The quantum information technology aims to achieve performance in communication and computation systems superior to those based on classical physics by utilizing the nonlocal quantum correlations of entangled states. It has also significantly improved understanding of the quantum systems involved on the factual realization of a quantum computer, and raised many interesting problems such as in the encoding of information 1 , entanglement of states $A$, quantum cryptography, quantum information manipulationt, and quantum communication of teleportation $⿴ 囗 ⿱ 一 一)$. One of the most striking features of quantum information is the quantum teleportations. In the quantum teleportation scheme, quantum information of an unknown 
state is faithfully transmitted from a sender to a remote receiver via an initially shared EPR pairs which function as a quantum information channel for the faithful transmission. Quantum teleportation was originally proposed for discrete variable in finite-dimensional Hilbert space, later it has been successfully developed from a discrete quantum system into a quantum system for continuous variables 6 . Teleportation of optical fields holds great promise due to the power of the required optical tools and the maturity of relevant optical communications technology. The quantum teleportation represents the basic building block of future quantum communication networks between distant parties 10 .

To enhance the performance of quantum teleportation system, some attempts have been made. One of them is to teleport a quantum state from sender to either of two receivers using the three-particle entanglement at certain condition of measurement 11 . Because of the experimental difficulties for generating the multiparticle entanglement state 12 , this teleportation scheme has not been implemented in experiments. In this paper, we proposed an new novel scheme to teleport an quantum state at Alice to different two receivers in turn using a two-mode squeezed state as the quantum switching to manipulate the transmission route. In this scheme, the EPR entangled beams shared by Alices and Bobs are produced by mixing a pair of two-mode squeezed state lights with identical frequency and constant phase relation on two beamsplitters of $50 \%$. As the usual teleportation scheme, one performs a certain joint measurement on the unknown input quantum state and one of EPR beams from the beamsplitters, and the Alice's measurement results are transmitted to two BOB's receivers by the classical lines, then that information is used to transform the second half of the EPR beams into an output state. Converting one of the two-mode squeezed state light between amplitude squeezing and phase squeezing, that can be easily realized by converting the relative phase of the pump field and injected field of OPA berween 0 and $\pi / 2[3$, the original unknown input quantum state can be conditionally mimiced at either of two Bobs in turn, so that the two-mode squeezed state light plays a role of quantum switching between two spatially separated receivers.

The scheme of the proposed system is shown in Fig.1. A pair of two-mode squeezed state 
light are used as the two EPR sources(EPR1 and EPR2). In the Heisenberg representation, the quadrature amplitude and phase operators $\hat{X}$ and $\hat{Y}$ for two modes of each two-mode squeezed state are expressed as follows 12,14 :

$$
\begin{aligned}
& \hat{X}_{a 1}=\left(e^{r_{a}} \hat{X}_{a 1}^{(0)}+e^{-r_{a}} \hat{X}_{a 2}^{(0)}\right) / \sqrt{2}, \hat{Y}_{a 1}=\left(e^{-r_{a}} \hat{Y}_{a 1}^{(0)}+e^{r_{a}} \hat{Y}_{a 2}^{(0)}\right) / \sqrt{2} \\
& \hat{X}_{a 2}=\left(e^{r_{a}} \hat{X}_{a 1}^{(0)}-e^{-r_{a}} \hat{X}_{a 2}^{(0)}\right) / \sqrt{2}, \hat{Y}_{a 2}=\left(e^{-r_{a}} \hat{Y}_{a 1}^{(0)}-e^{r_{a}} \hat{Y}_{a 2}^{(0)}\right) / \sqrt{2} \\
& \hat{X}_{b 1}=\left(e^{r_{b}} \hat{X}_{b 1}^{(0)}+e^{-r_{b}} \hat{X}_{b 2}^{(0)}\right) / \sqrt{2}, \hat{Y}_{b 1}=\left(e^{-r_{b}} \hat{Y}_{b 1}^{(0)}+e^{r_{b}} \hat{Y}_{b 2}^{(0)}\right) / \sqrt{2} \\
& \hat{X}_{b 2}=\left(e^{r_{b}} \hat{X}_{b 1}^{(0)}-e^{-r_{b}} \hat{X}_{b 2}^{(0)}\right) / \sqrt{2}, \hat{Y}_{b 2}=\left(e^{-r_{b}} \hat{Y}_{b 1}^{(0)}-e^{r_{b}} \hat{Y}_{b 2}^{(0)}\right) / \sqrt{2}
\end{aligned}
$$

where the subscripts $a_{1}, a_{2}$ and $b_{1}, b_{2}$ are designated for the two modes in the two-mode squeezed state from EPR1 and EPR2 respectively. The subscript "(0)" denotes initial coherent modes, $r_{a}$ and $r_{b}$ are the correlation parameters between $a_{1}$ and $a_{2}$ as well as $b_{1}$ and $b_{2}$ mode. Under the limit of perfect correlation $r_{a} \rightarrow \infty$ and $r_{b} \rightarrow \infty$, the two twomode squeezed states approach the eigenstates of $\hat{X}_{a(b) 1}-\hat{X}_{a(b) 2}$ and $\hat{Y}_{a(b) 1}+\hat{Y}_{a(b) 2}$, which corresponding to two perfect EPR pairs having the quadrature amplitude correlation and quadrature phase anticorrelation. Otherwise, if $r_{a} \rightarrow-\infty$ and $r_{b} \rightarrow-\infty$, it approach the eigenstates of $\hat{X}_{a(b) 1}+\hat{X}_{a(b) 2}$ and $\hat{Y}_{a(b) 1}-\hat{Y}_{a(b) 2}$, which are also the perfect EPR state with the anticorrelated quadrature amplitude and correlated quadratures phase.

In order to perform a teleportation switching, the sender has to share simultaneously the entanglement with two receivers. Initially, mode $a_{1}$ shares the entanglement with the mode $a_{2}$ and mode $b_{1}$ shares the entanglement with the mode $b_{2}$, then we mix the mode $a_{1}$ and $a_{2}$ with the mode $b_{1}$ and $b_{2}$ at the beamsplitters BS1 and BS2 respectively. The output modes of the two beamsplitters are:

$$
\begin{aligned}
& \hat{X}_{3}=\left(\hat{X}_{a 1}-\hat{X}_{b 1}\right) / \sqrt{2}, \hat{Y}_{3}=\left(\hat{Y}_{a 1}-\hat{Y}_{b 1}\right) / \sqrt{2} \\
& \hat{X}_{4}=\left(\hat{X}_{a 1}+\hat{X}_{b 1}\right) / \sqrt{2}, \hat{Y}_{4}=\left(\hat{Y}_{a 1}+\hat{Y}_{b 1}\right) / \sqrt{2} \\
& \hat{X}_{5}=\left(\hat{X}_{a 2}+\hat{X}_{b 2}\right) / \sqrt{2}, \hat{Y}_{5}=\left(\hat{Y}_{a 2}+\hat{Y}_{b 2}\right) / \sqrt{2} \\
& \hat{X}_{6}=\left(\hat{X}_{a 2}-\hat{X}_{b 2}\right) / \sqrt{2}, \hat{Y}_{4}=\left(\hat{Y}_{a 2}-\hat{Y}_{b 2}\right) / \sqrt{2} .
\end{aligned}
$$

Therefore we have: 


$$
\begin{aligned}
\hat{X}_{3}-\hat{X}_{5} & =\left[\left(\hat{X}_{a 1}-\hat{X}_{a 2}\right)-\left(\hat{X}_{b 1}+\hat{X}_{b 2}\right)\right] / \sqrt{2} \\
\hat{Y}_{3}+\hat{Y}_{5} & =\left[\left(\hat{Y}_{a 1}+\hat{Y}_{a 2}\right)-\left(\hat{Y}_{b 1}-\hat{Y}_{b 2}\right)\right] / \sqrt{2} \\
\hat{X}_{3}-\hat{X}_{6} & =\left[\left(\hat{X}_{a 1}-\hat{X}_{a 2}\right)-\left(\hat{X}_{b 1}-\hat{X}_{b 2}\right)\right] / \sqrt{2} \\
\hat{Y}_{3}+\hat{Y}_{6} & =\left[\left(\hat{Y}_{a 1}+\hat{Y}_{a 2}\right)-\left(\hat{Y}_{b 1}+\hat{Y}_{b 2}\right)\right] / \sqrt{2} .
\end{aligned}
$$

It is obviously that the mode $a_{3}$ will be entangled with the mode $a_{5}$ if one of the twomode squeezed states is the eigenstate of $\hat{X}_{a 1}-\hat{X}_{a 2}$ and $\hat{Y}_{a 1}+\hat{Y}_{a 2}\left(\right.$ i.e. $\left.r_{a} \rightarrow \infty\right)$, and the other one is the eigenstate of $\hat{X}_{b 1}+\hat{X}_{b 2}$ and $\hat{Y}_{b 1}-\hat{Y}_{b 2}$ (i.e. $\left.r_{b} \rightarrow-\infty\right)$. The mode $a_{3}$ will be entangled with the mode $a_{6}$ at the conditions of $r_{a} \rightarrow \infty, r_{b} \rightarrow \infty$ or $r_{a} \rightarrow-\infty, r_{b} \rightarrow-\infty$. According to the different entanglement condition the quantum information from a sender can be controllably transmitted to Bob1 or Bob2.

In Alice's station an unknown input quantum state represented by the quadrature operators $\hat{X}_{i n}$ and $\hat{Y}_{i n}$ is superposed with $a_{3}$ mode at the beamsplitter BS3. Two balanced homodyne detectors $D_{X c}$ and $D_{Y c}$ are used to measure the observables of the quadrature amplitude $\hat{X}_{c}=\left(\hat{X}_{i n}-\hat{X}_{3}\right) / \sqrt{2}$ of one output of BS3 and the quadrature phase $\hat{Y}_{c}=\left(\hat{Y}_{\text {in }}+\hat{Y}_{3}\right) / \sqrt{2}$ of the other output of BS3. The resulting classical outcomes are scaled by the operators:

$$
\begin{aligned}
& \hat{X}_{c}=\left[\hat{X}_{i n}-\left(\hat{X}_{a 1}-\hat{X}_{b 1}\right) / \sqrt{2}\right] / \sqrt{2} \\
& \hat{Y}_{c}=\left[\hat{Y}_{i n}+\left(\hat{Y}_{a 1}-\hat{Y}_{b 1}\right) / \sqrt{2}\right] / \sqrt{2}
\end{aligned}
$$

Due to the entanglement between the modes $a_{3}$ and $a_{5}$, or $a_{3}$ and $a_{6}$ at different conditions, the measurements lead to that the mode $a_{5}$ or $a_{6}$ collapses into a state which differs from the unknown input state in the classical phase-space displacement. Thus for the possibility to recover the input state at the two locations, each of the classical outcomes is divided into two same parts with RF power splitters $(\mathrm{RF} 1$ and RF2) 10 , and the classical information is sent separately to the two remote locations for performing appropriate displacements on modes $\hat{a}_{5}$ and $\hat{a}_{6}$ :

$$
\begin{aligned}
& \hat{a}_{5} \rightarrow \hat{a}_{5}^{\text {out }}=\hat{a}_{5}+\sqrt{2} g_{1} \hat{a}_{c} \\
& \hat{a}_{6} \rightarrow \hat{a}_{6}^{\text {out }}=\hat{a}_{6}+\sqrt{2} g_{2} \hat{a}_{c},
\end{aligned}
$$


where $\hat{a}_{c}$ represents the annihilation operator of coherent state generating from the classical results $\hat{a}_{c}=\hat{X}_{c}+i \hat{Y}_{c}$.

According to Eqs.(1) (2) (4) and (5), the outgoings of two modes become:

$$
\begin{aligned}
\hat{a}_{5}^{\text {out }}= & g_{1} \hat{a}_{i n}+\frac{1+g}{2}\left[e^{-r_{a}}\left(-\hat{X}_{a 2}^{(0)}+i \hat{Y}_{a 1}^{(0)}\right)+e^{r_{b}}\left(\hat{X}_{b 1}^{(0)}-i \hat{Y}_{b 2}^{(0)}\right)\right] \\
& +\frac{1-g}{2}\left[e^{r_{a}}\left(\hat{X}_{a 1}^{(0)}-i \hat{Y}_{a 2}^{(0)}\right)+e^{-r_{b}}\left(-\hat{X}_{b 2}^{(0)}+i \hat{Y}_{b 1}^{(0)}\right)\right] \\
\hat{a}_{6}^{\text {out }}= & g_{2} \hat{a}_{i n}+\frac{1+g}{2}\left[e^{-r_{a}}\left(-\hat{X}_{a 2}^{(0)}+i \hat{Y}_{a 1}^{(0)}\right)+e^{-r_{b}}\left(\hat{X}_{b 2}^{(0)}-i \hat{Y}_{b 1}^{(0)}\right)\right] \\
& \frac{1-g}{2}\left[e^{r_{a}}\left(\hat{X}_{a 1}^{(0)}-i \hat{Y}_{a 2}^{(0)}\right)+e^{r_{b}}\left(-\hat{X}_{b 1}^{(0)}+i \hat{Y}_{b 2}^{(0)}\right)\right],
\end{aligned}
$$

where the parameters $g_{1}$ and $g_{2}$ describe the normalized gain of two teleportation processes from a sender to Bob1 and Bob2.

The $\operatorname{Eq}(6)$ show that both the output modes $\hat{a}_{5}^{\text {out }}$ and $\hat{a}_{6}^{\text {out }}$ contain some information about the teleported state but it is not exactly the input state due to some additional noise from the quantum channels.

For the case of ideal measurement process $g_{1}=1$ and $r_{a} \rightarrow \infty, r_{b} \rightarrow-\infty$ (or $r_{a} \rightarrow$ $\left.-\infty, r_{b} \rightarrow \infty\right)$. The $\operatorname{Eq}(6)$ becomes $\hat{a}_{5}^{\text {out }}=\hat{a}_{i n}$. So the perfect quantum teleportation is accomplished at the receiver Bob1.

If $g_{2}=1$ and $r_{a} \rightarrow \infty, r_{b} \rightarrow \infty$ (or $r_{a} \rightarrow-\infty, r_{b} \rightarrow-\infty$ ), we have $\hat{a}_{6}^{\text {out }}=\hat{a}_{i n}$, then the unknown quantum state is perfectly mimiced at the receiver Bob2.

The fidelity quantifying the quality of teleportation is defined for a coherent input state by 16 .17:

$$
\begin{aligned}
F= & \frac{2}{\sqrt{\left(\left\langle\delta^{2} \hat{X}_{\text {out }}\right\rangle+1\right)\left(\left\langle\delta^{2} \hat{Y}_{\text {out }}\right\rangle+1\right)}} \\
& \exp \left[-2 \frac{(1-g)^{2}\left|\alpha_{\text {in }}\right|^{2}}{\sqrt{\left(\left\langle\delta^{2} \hat{X}_{\text {out }}\right\rangle+1\right)\left(\left\langle\delta^{2} \hat{Y}_{\text {out }}\right\rangle+1\right)}}\right],
\end{aligned}
$$

where $\delta^{2} \hat{X}_{\text {out }}$ and $\delta^{2} \hat{Y}_{\text {out }}$ are the variance of quadrature amplitude and phase of the output mode. Using Eqs(6), they are given by:

$$
\left\langle\delta^{2} \hat{X}_{\text {out }}\right\rangle=g_{1(2)}^{2}\left\langle\delta^{2} \hat{X}_{i n}\right\rangle+\left(\frac{1+g}{2}\right)^{2}\left[e^{-2 r_{a}}+e^{ \pm 2 r_{b}}\right]+\left(\frac{1-g}{2}\right)^{2}\left[e^{2 r_{a}}+e^{\mp 2 r_{b}}\right]
$$




$$
\left\langle\delta^{2} \hat{Y}_{\text {out }}\right\rangle=g_{1(2)}^{2}\left\langle\delta^{2} \hat{Y}_{\text {in }}\right\rangle+\left(\frac{1+g}{2}\right)^{2}\left[e^{-2 r_{a}}+e^{ \pm 2 r_{b}}\right]+\left(\frac{1-g}{2}\right)^{2}\left[e^{2 r_{a}}+e^{\mp 2 r_{b}}\right]
$$

in Eq.(8), the symbol " \pm " and "1(2)" represent that the teleportation is accomplished at the output mode of $\hat{a}_{5}$ or $\hat{a}_{6}$ respectively.

In the classical system without quantum correlation $r_{a}=0$ and $r_{b}=0$, we obtain $F=1 / 2$ for the normalized gain $g_{1(2)}=1$, so the classical limit of teleportation in this system keeps the same with the usual teleportation system for continuous variables 17 . According to eq.(7), to meet the requirement of the quantum teleportation $F>1 / 2$, only requires that either of the two the initial light fields from EPR1 and EPR2 is a two-mode squeezed state, even while the other one is a coherent state light. But if one wants to get high fidelity two of them should have high squeezing.

For experiments the most important work is to establish two EPR beam sources with identical frequency and constant phase relation. Two same degenerate ${ }^{\mathrm{\theta}}$ or nondegenerate optical parametric amplifiers 18 . 19 (DOPA or NOPA) pumped by a same laser can be used to produce the needed two two-mode squeezed states. The mature parametric technique is beneficial to complete the proposed prototype. The correlation relation between two modes of EPR pairs can be manipulated by converting the relative phase between pump field and injected signal field of OPA between 0 and $\pi$. For the parametric deamplification (the pump field and the injected field are in phase of 0), the two-mode amplitude squeezing is completed which corresponding to the EPR beams with the quadrature amplitude correlation and quadrature phase anticorrelation between two modesed. And for a polarization nondegenerate parametric amplification( the pump field and the injected field are out of phase i.e. the relative phase is $\pi$ ), the two-mode phase squeezing is obtained which corresponding to the quadrature amplitude anticorrelation and quadrature phase correlation EPR beams 8 .192122.

In conclusion, we propose an quantum switching system for sending controllably an unknown quantum state to either of two remote receivers. The control condition is only to convert the squeezed component of one of two two-mode squeezed states between its 
quadrature amplitude and phase. The conditional teleportation system might be developed as a practical quantum switching in future quantum communication. The well-known optical parametric technique provides great convenience for its experimental demonstration.

\section{ACKNOWLEDGMENTS}

This research is supported by the National Natural Science Foundation of China (No.69978013) and the Oversea Youth Scholar Collabration Foundation(No.69928504).

1. J.Preskill, e-print quant-ph/9904022.

2. A.Zeilinger, Phys.World 11, 35(1998).

3. W.Tittel, G.Ribordy and N.Gisin, Phys.World 11, 41(1998).

4. M.A.Nielsen, Phys.Re.Lett., 83, 436(1999).

5. C.H.Bennett, G.Brassard, C.Crepeau, Phys.Rev.Lett., 70, 1895(1993).

6. D.Bouwmeester, J.W.Pan, K.Mattle, M.Edibl, H.Weinfurter and A.Zeilinger, Nature, 390, $575(1997)$.

7. D.Boschi, S.Branca, F.De Martini, L.Hardy and S.Popescu, Phys.Rev.Lett., 80, 1121(1998).

8. L.Vaidman, Phys.Rev.A49, 1473(1994); S.L.Braunstein, H.J.Kimble, Phys.Rev.Lett., 80, 869(1998).

9. A.Furusawa, J.L.Sorensen, S.L.Braunstein, C.A.Fuchs, H.J.Kimble, E.S.Polzik, Science, 282, 706(1998).

10. S.Bose, V.Vedral and P.L.Knight, Phys.Rev.A57, 822(1998).

11. A.Karlsson and M.Bourennane, Phys.Rev.A, 58, 4394(1998).

12. P.van Loock and S.L.Braunstein, Phys.Rev.Lett., 84, 3482(2000). 
13. S.F.Pereira, Z.Y.Ou and H.J.Kimble, Phys.Rev.A, 62, 042311(2000).

14. D.F.Walls and G.J.Milburn, Quantum Optics(Springer Verlag), Berlin, 1994.

15. J.Zhang, K.C.Peng, will be printed in PRA in November.

16. S.L.Braunstein, C.A.Fuchs, and H.J.Kimble, J.Mod.Opt., 47,267(2000); S.L.Braunstein, C.A.Fuchs, and H.J.Kimble, e-print, quant-ph/9910030.

17. R.E.S.Polkinghorne and T.C.Ralph, Phys.Rev.Lett., 83, 2095(1999).

18. Z.Y.Ou, S.F.Pereira, H.J.Kimble, and K.C.Peng, Phys.Rev.Lett., 68, 3663(1992).

19. Y.Zhang, H.Wang, X.Y.Li, J.T.Jing, C.D.Xie, K.C.Peng, Phys.Rev.A, 62, 023813(2000).

20. Y.Zhang, H.Su, C.D.Xie, K.C.Peng, Phys.Lett.A, 259, 171(1999).

21. K.Schneider, R.Bruckmeier, H.Hansen, S.Schiller and J.Mlynek, Opt.Lett., 21, 1396(1996).

22. Y.Aharonov et al., Ann.Phys.(N.Y.), 39, 498(1966). 